\title{
Instability in internal solitary waves with trapped cores
}

\author{
M. Carr*, S. E. King and D. G. Dritschel \\ School of Mathematics \& Statistics, University of St Andrews, \\ St Andrews, KY16 9SS, U.K. \\ * Corresponding author, email: magda@mcs.st-and.ac.uk, \\ Phone:+44(1334)463715, Fax:+44(1334)463748
}

December 8, 2011

\begin{abstract}
A numerical method that employs a combination of contour advection and pseudospectral techniques is used to investigate instability in internal solitary waves with trapped cores. A three-layer configuration for the background stratification in which the top two layers are linearly stratified and the lower layer is homogeneous is considered throughout. The strength of the stratification in the very top layer is chosen to be sufficient so that waves of depression with trapped cores can be generated. The flow is assumed to satisfy the Dubriel-Jacotin-Long equation both inside and outside of the core region. The Brunt-Väisälä frequency is modelled such that it varies from a constant value outside of the core to zero inside the core over a sharp but continuous transition length. This results in a stagnant core in which the vorticity is zero and the density is homogeneous and approximately equal to that at the core boundary. The time dependent simulations show that instability occurs on the boundary of the core. The instability takes the form of Kelvin-Helmholtz billows. If the instability in the vorticity field is energetic enough disturbance in the buoyancy field is also seen and fluid
\end{abstract}


exchange takes place across the core boundary. Occurrence of the Kelvin-Helmholtz billows is attributed to the sharp change in the vorticity field at the boundary between the core and the pycnocline. The numerical scheme is not limited by small Richardson number unlike the other alternatives currently available in the literature which appear to be.

Keywords: Internal Solitary Waves, Trapped Cores.

\section{INTRODUCTION}

Internal solitary waves (ISWs) are ubiquitous features in the Earth's atmosphere and ocean. ${ }^{1-4}$ In the open ocean, typically, the waves are highly nonlinear and may attain very large amplitudes. $^{5-7}$ It is well known that at such large amplitudes, ISWs of depression (elevation) may exhibit trapped cores if the density gradient at the surface (bottom) of the water column is finite (and waves are supported in which the local horizontal fluid velocity exceeds the wave speed). As well as in the ocean, ${ }^{8,9}$ waves of this type have been observed in the atmosphere, ${ }^{10-12}$ the laboratory, ${ }^{13,14}$ and in theoretical studies. ${ }^{15-25}$ Despite this a physically consistent theory for waves with trapped cores remains elusive and there is debate in the literature as to how ISWs with trapped cores should be modelled.

The most common approach has been to model the flow, both inside and outside of the core region, using the Dubriel-Jacotin-Long ${ }^{26,27}$ (DJL) equation

$$
\nabla^{2} \eta+\frac{N^{2}(Y)}{c^{2}} \eta=0
$$

where $\eta(x, y)$ is the departure of a streamline from its background vertical position, $Y=y-\eta$, $N$ is the background Brunt-Väisälä frequency and $c$ is the wave speed. In such models the manner in which the Brunt-Väisälä frequency is extended in the core region is arbitrary since by definition no streamlines in the core connect upstream of the wave. The majority 
of models have assumed that the Brunt-Väisälä frequency in the core region takes the same value as that at the core boundary, i.e. the stratification is simply extended into the core region. This idea goes back to Davis \& $\operatorname{Acrivos}^{15}$ and examples of it can be found in Brown \& Christie $^{17}$ and Fructus \& Grue. ${ }^{22}$ However, models of this form lead to solutions in which the core density is statically unstable and hence are physically unrealizable (both Brown \& Christie $^{17}$ and Fructus \& Grue ${ }^{22}$ note this). Motivated by field observations, King et al. ${ }^{24}$ assume that the Brunt-Väisälä frequency varies from a constant outside of the core to zero inside the core over a sharp but continuous transition length. This results in a stagnant (zero vorticity) core in the wave frame of reference, of constant density where the density closely matches that on the core boundary. Using a fully nonlinear time dependent code, King et al. ${ }^{24}$ show that the stagnant core model, at an amplitude just after core formation, is essentially stable. Small-scale disturbances are seen along the core boundary and are attributed to numerical fringing. In this paper, a wider parameter space than in King et al. ${ }^{24}$ is considered and it is shown that the small-scale disturbances seen in King et al. ${ }^{24}$ are more likely to be due to Kelvin-Helmholtz instability on the core boundary than numerical fringing. It is shown, therefore, that the steady state solutions of King et al., ${ }^{24}$ are weakly unstable or unstable (the degree of instability essentially being dependent on the wave amplitude).

Assuming that the DJL equation holds within the core region is mathematically acceptable but has no physical justification, Derzho \& Grimshaw ${ }^{16}$ suggest an alternative is to model the flow within the core using a vorticity-streamfunction relation and to match it to the flow outside the core through a pressure continuity condition at the core boundary. Derzho \& Grimshaw ${ }^{16}$ originally did this for a linear background stratification and recently Helfrich \& White ${ }^{23}$ extended the model for an arbitrary stratification. In Derzho \& Grimshaw, ${ }^{16}$ the core is shown to have zero vorticity to leading order. This was subsequently confirmed numerically by Aigner et al. ${ }^{18}$ In principle, the vorticity-streamfunction 
relation and the density in the core are arbitrary. To make progress, Helfrich \& White ${ }^{23}$ concentrated on the simplest assumption, namely, that the core had zero vorticity and the density in the core was equal to that at the core boundary. Evolution of their model, at an amplitude just after core formation, resulted in small disturbances being seen on the core boundary. With time the wave restablized and took approximately the same form as the initial (steady state) wave. At higher amplitudes, however, Kelvin-Helmholtz instability was seen along the core boundary which did not diminish with time. Without exception, all steady state models of ISWs with trapped cores in the literature, that are evolved through time-dependent simulations result in weakly unstable or unstable waves. ${ }^{18,19,23-25}$

In the laboratory, both Grue et al. ${ }^{13}$ and Carr et al. ${ }^{14}$ have observed unstable ISWs with trapped cores. Grue et al. ${ }^{13}$ observed trapped cores in which small vortices took place in the leading part of the wave. In Carr et al. ${ }^{14}$ larger amplitudes than in Grue et al. ${ }^{13}$ were considered and in addition to observing small scale vortices, shear instability was seen. No laboratory evidence of stable (mode one) ISWs with trapped cores has been presented. Hence all numerical and laboratory studies to date seem to suggest that ISWs with trapped cores are inherently unstable. Unfortunately, it is not possible to check this conjecture from presently available field data ${ }^{8-12}$ due to a lack of resolution in the core measurements.

Waves with trapped cores provide a very effective transport mechanism and are thought to play an important role in cross-shore larvae transport. ${ }^{28,29}$ In addition, unstable ISWs with trapped cores are expected to be an important source of mixing, turbulence, and redistribution of potential energy in the water column. To understand the behavior of unstable ISWs with trapped cores, it is imperative that the evolutionary processes which lead to breaking and the subsequent generation of turbulence be better understood. In this paper the unstable evolution of an ISW initially given via the steady state model of King et al. ${ }^{24}$ is investigated. Results are compared with those from the stagnant core model of Helfrich \& White $^{23}$ and it is found that the two models are qualitatively equivalent. Note that the model 
presented in Helfrich \& White ${ }^{23}$ uses the variational formulation proposed by Turkington et al. ${ }^{30}$ to find a solution to the DJL equation and as such appears to be limited by small Richardson number. ${ }^{20}$ The model considered here provides an alternative (but qualitatively similar) means of modelling ISWs with trapped cores that is not limited by small Richardson numbers. Note that recently, Lamb \& Farmer ${ }^{31}$ have computed ISWs using a method based on Turkington et al.'s ${ }^{30}$ formulation that does not appear to be limited by small Richardson number.

The paper is laid out at follows. In Sec. II the governing equations are given, in Sec. III the numerical method is explained and in Sec. IV numerical results are presented including a comparison with the stagnant core model of Helfrich \& White. ${ }^{23}$ Finally in Sec. V some conclusions are made.

\section{GOVERNING EQUATIONS}

To model the time dependent motion of an ISW the inviscid, incompressible, OberbeckBoussinesq equations in two dimensions are used:

$$
\begin{aligned}
\rho_{0}\left(\mathbf{u}_{t}+\mathbf{u} \cdot \nabla \mathbf{u}\right) & =-\nabla p-\rho g \mathbf{j} \\
\rho_{t}+\mathbf{u} \cdot \nabla \rho & =0 \\
\nabla \cdot \mathbf{u} & =0
\end{aligned}
$$

where $\rho_{0}$ is a convenient constant reference density, $\mathbf{u}=(u, v)$ is the fluid velocity vector, $t$ denotes time, $\nabla=(\partial / \partial x, \partial / \partial y)$ is the gradient operator, $p$ is the fluid pressure, $\rho$ is the fluid density, $g$ is the acceleration due to gravity and $\mathbf{j}$ is the unit vector in the vertical direction. Buoyancy and vorticity are introduced as $b=-g\left(\rho-\rho_{0}\right) / \rho_{0}$ and $\zeta=v_{x}-u_{y}$, respectively. Then taking the curl of the momentum equation (1) and rewriting (1) and (2) in terms of 


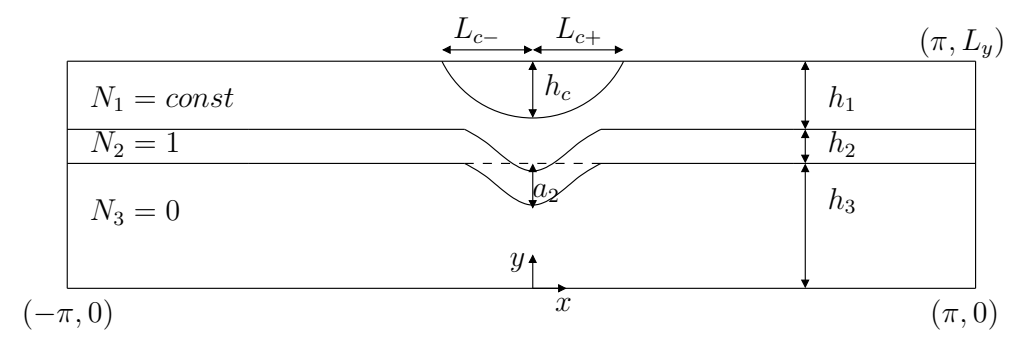

Figure 1: A schematic diagram of the computational domain (not to scale).

vorticity and buoyancy gives

$$
\begin{aligned}
\zeta_{t}+\mathbf{u} \cdot \nabla \zeta & =b_{x}, \\
b_{t}+\mathbf{u} \cdot \nabla b & =0, \\
\nabla \cdot \mathbf{u} & =0 .
\end{aligned}
$$

To study the evolution of an ISW satisfying (4)-(6) two numerical schemes are employed. The first, which is outlined in detail in King et al. ${ }^{24}$ and briefly in Sec. III B, is an iterative procedure which finds a steady state solution. The second, which is outlined in detail in Dritschel \& Fontane ${ }^{32}$ and briefly in Sec. III D, is the Combined Lagrangian Advection Method (CLAM) which takes a steady state solution as input and evolves it with time.

\section{NUMERICAL METHOD}

\section{A. Numerical Setup}

A schematic diagram of the computational domain is given in figure 1 . The domain is chosen to be $2 \pi$ periodic in the horizontal $x$ direction and bounded above and below by rigid boundaries at $y=0$ and $y=L_{y}$. The aspect ratio of the domain, $L_{y} / 2 \pi$, is chosen to be 0.1 throughout the paper. This ensures that the domain is long compared to the length of the waves. Note that an aspect ratio of 0.05 was also investigated and entirely analogous results 
were found. A three layer stratification is considered in which the thicknesses of the top, middle and bottom layers are denoted by $h_{1}, h_{2}$ and $h_{3}$ respectively. Attention is restricted to modelling ISWs of depression, i.e. $h_{3}>\left(h_{1}+h_{2}\right)$. Waves of elevation can be obtained by symmetry for $h_{3}<\left(h_{1}+h_{2}\right)$. The domain is chosen so that the ISW is located in the center of the domain as shown. The maximum displacement of the interface between the middle and bottom layers is denoted by $a_{2}$. The undisturbed Brunt-Väisälä frequency, $N$, is defined by

$$
N^{2}(Y)=\frac{\mathrm{d} \bar{b}}{\mathrm{~d} Y}=-\frac{g}{\rho_{0}} \frac{\mathrm{d} \bar{\rho}}{\mathrm{d} Y}
$$

where $Y$ is an isopycnal coordinate used to denote $y$ in the far field and bars denote background (undisturbed) variables. The undisturbed Brunt-Väisälä frequency is assumed to be zero in the bottom layer $\left(N_{3}=0\right)$, unity in the middle layer $\left(N_{2}=1\right)$, a given constant in the top layer $\left(N_{1}\right)$ and to have a smooth transition between these values such that

$$
N^{2}(Y)=\frac{N_{1}^{2}}{2}+\frac{1}{2} \operatorname{erf}\left(\frac{Y-h_{3}}{\delta}\right)+\frac{N_{1}^{2}-1}{2} \operatorname{erf}\left(\frac{Y-\left(h_{2}+h_{3}\right)}{\delta}\right),
$$

where erf denotes the error function and $\delta$ represents a distance over which the profile is smoothed. Throughout the paper, a value of two $y$ grid lengths is chosen for $\delta$ and a resolution of $\left(n_{x}, n_{y}\right)=(1024,128)$, where $n_{x}$ and $n_{y}$ are the number of grid points in the horizontal and vertical directions is used. Justification of these choices and the effect of varying $\delta$ or $\left(n_{x}, n_{y}\right)$ in a similar configuration is given in King et al. ${ }^{24}$ and Carr et al. ${ }^{35}$

\section{B. The steady state solver}

To find a steady state solution, the numerical scheme of King et al. ${ }^{24}$ is employed. Following Yih $^{36}$ and Grue et al. ${ }^{13}$ it was shown in King et al. ${ }^{24}$ that (1) (or equivalently (4)) can be 
rewritten as the so called Dubriel-Jacotin-Long (DJL) equation ${ }^{26,27}$

$$
\zeta=\nabla^{2} \psi=-\frac{N^{2}(Y)}{c^{2}} \psi
$$

where $\psi(x, y)$ is the streamfunction, defined such that $u=-\psi_{y}$, and $v=\psi_{x}$. Solutions of (8) for a given $N^{2}(Y)$ are computed using an iterative procedure. First, a uniform computational grid is set up within the domain. The background buoyancy field, $\bar{b}(Y)$, is then found by integrating a given profile of $N^{2}(Y)$ with respect to $Y$. The iterative solution procedure is then started with a guess for $\psi$. A weakly nonlinear solitary wave solution is used for this purpose which is known to be accurate at small wave amplitudes. The wave amplitude is defined as $\mathcal{A}=\eta_{r m s}=\psi_{r m s} / c$, where $\eta$ is the (downward) streamline displacement and $r m s$ denotes the root-mean-square value. By using the wave speed $c_{w n l}$ from the weakly nonlinear solution, ${ }^{24}$ an initial amplitude $\mathcal{A}_{0}=\psi_{r m s} / c_{w n l}$ is found. This initial guess is then corrected by solving

$$
Y=y+\frac{\psi(x, y)}{c}
$$

for the isopycnal coordinate $Y$ followed by (8) for $\zeta$ at each point in the domain (using the previous guess for $\psi$ in the right hand side of (8)). Spectral inversion of $\nabla^{2} \psi=\zeta$ provides an updated value for $\psi$, and

$$
c=\frac{\psi_{r m s}}{\mathcal{A}},
$$

provides an updated value for $c$. This process is then repeated, by solving (9), (8), and (10) until $\psi$ converges. ${ }^{24}$ Subsequent states are found for higher amplitudes by increasing $\mathcal{A}$ in increments of $\delta \mathcal{A}=0.002$.

Using the variational formulation proposed by Turkington et al., ${ }^{30}$ Lamb $^{20}$ showed that solutions of (8) can be found for increasing wave amplitude (and speed) until the solution ends in one of three outcomes, namely, (i) a low Richardson number shear instability limit, 
(ii) a flat-top wave, or conjugate state limit or (iii) a breaking limit defined by the presence of incipient overturning $(u=c)$. The numerical scheme outlined above does not appear to be limited by low Richardson numbers. ${ }^{24}$ The focus of this paper is to use the model presented in King et al. $^{24}$ on ISWs that are limited by incipient overturning. A necessary condition for incipient overturning in waves of depression (or elevation) is that $N^{2}$ is non-zero at the top (or bottom) of the fluid column. ${ }^{17,22-24}$ It is well known that closed streamlines (or core regions) occur in such flows.

\section{Closed streamline regions}

The numerical scheme outlined in Sec. III B relies on the existence of a streamline connecting any point within the domain to the upstream edge of the domain. This is problematic in cases where closed streamlines are present in the flow. To solve this problem King et al. ${ }^{24}$ followed previous authors ${ }^{17,22}$ and extended the definition of $Y$ by allowing $Y$ to exceed the domain height $L_{y}$ for closed streamlines. The way in which $N^{2}$ should be defined for values of $Y$ in this region is open to debate. Brown \& Christie $^{17}$ and Fructus \& Grue $^{22}$ (following an idea that goes back to Davis \& Acrivos $^{15}$ ) let

$$
N^{2}(Y)=N^{2}\left(L_{y}\right), \quad \text { for } \quad Y>L_{y}
$$

This leads to a core region of the flow containing a density field that is statically unstable and is therefore physically unrealizable (both Brown \& Christie $^{17}$ and Fructus \& Grue ${ }^{22}$ note this).

Field observations in the atmosphere and the ocean ${ }^{8-12}$ suggest that ISWs with trapped cores (closed streamlines) have constant density within the core. Motivated by this King et 
al. ${ }^{24}$ suggested a different extension for $N^{2}$ namely

$$
N^{2}(Y)=N^{2}\left(L_{y}\right) \exp \left(-\left(\left(Y-L_{y}\right) / 10^{-9} L_{y}\right)^{2}\right), \quad \text { for } \quad Y>L_{y} .
$$

In this case, $N^{2}$ varies from $N^{2}\left(L_{y}\right)$ to zero (constant density) over a sharp but continuous transition length in the core region. This results in the core region having a constant density approximately equal to that at the top of the domain. Brown \& Christie, ${ }^{17}$ Fructus \& Grue $^{22}$ and King et al. ${ }^{24}$ all assumed that the DJL equation (8) holds in the core region and the vorticity field in the core is given directly from the respective definitions of $N^{2}$ in that region. The model given by (11) results in a core with constant non-zero vorticity and a statically unstable density field while (12) results in a stagnant core in which the vorticity is zero and the density is homogeneous and approximately equal to that at the core boundary. However, there is no physical justification for using the DJL equation (8) in the core region (refs ${ }^{17,22,24}$ all point this out). An alternative approach is to model the flow within the core using a vorticity-streamfunction relation and to match it to the flow outside the core through a pressure continuity condition at the core boundary. Derzho \& Grimshaw ${ }^{16}$ originally did this for a linear background stratification and recently Helfrich \& White ${ }^{23}$ extended the model for an arbitrary stratification. In principle, the vorticity-streamfunction relation and the density in the core are arbitrary. To make progress, in the majority of their analysis, Helfrich \& White, ${ }^{23}$ made the simplest assumption, namely, that the core has zero vorticity and the density in the core is equal to that at the core boundary. King et al. ${ }^{24}$ presented a third alternative to modeling the core which resulted in the vorticity being a non-zero constant in the core. Evolution of this mathematical steady state resulted in an unsteady wave with a rotating core. King et al. ${ }^{24}$ also presented the evolution of steady states which were modeled using the DJL equation in the core region with $N^{2}$ either of the form (11) (non-zero vorticity in core region) or (12) (zero vorticity in core region). The model given by 
(12) (zero vorticity in core region) was substantially more stable than the approach given by

(11) (non-zero vorticity in core region) and the alternative constant vorticity rotating core model. Hence in this paper the zero vorticity stagnant core model (12) is focused upon and a comparison with the model of Helfrich \& White ${ }^{23}$ is given in Sec. IV.

\section{The unsteady solver}

To study the evolution of a steady state satisfying (4)-(6) the numerical procedure outlined in Dritschel \& Fontane ${ }^{32}$ is utilized. A hyperdiffusive vorticity term $\nu_{6} \nabla^{6} \zeta$ (with diffusion coefficient $\nu_{6}=2^{-54}$ ) is added to the right hand side of (4) in order to stabilize the solution. The unsteady simulations are then carried out using CLAM, an algorithm which uses a combination of contour advection ${ }^{37}$ and pseudo-spectral techniques in order to integrate (5) forward in time. This provides an accurate source term for the right hand side of (4), which is solved using a pseudo-spectral method. Temporal integration is performed using a fourth order Runge-Kutta integration scheme. Further details of the method can be found in Dritschel \& Scott, ${ }^{33}$ Fontane \& Dritschel ${ }^{34}$ and Dritschel \& Fontane. ${ }^{32}$

\section{NUMERICAL RESULTS}

\section{A. Steady State Core Characteristics}

In this section results from the steady state code are presented. Attention will focus upon cases in which the Brunt-Väisälä frequency in the top layer $N_{1}$ is finite so that a region with closed streamlines can occur in the top of the domain. Closed streamline regions are modeled via equation (12).

In figure 2, a plot of wave amplitude versus wave speed for varying lower layer thickness is given. The undisturbed background stratification is such that the Brunt-Väisälä frequency 


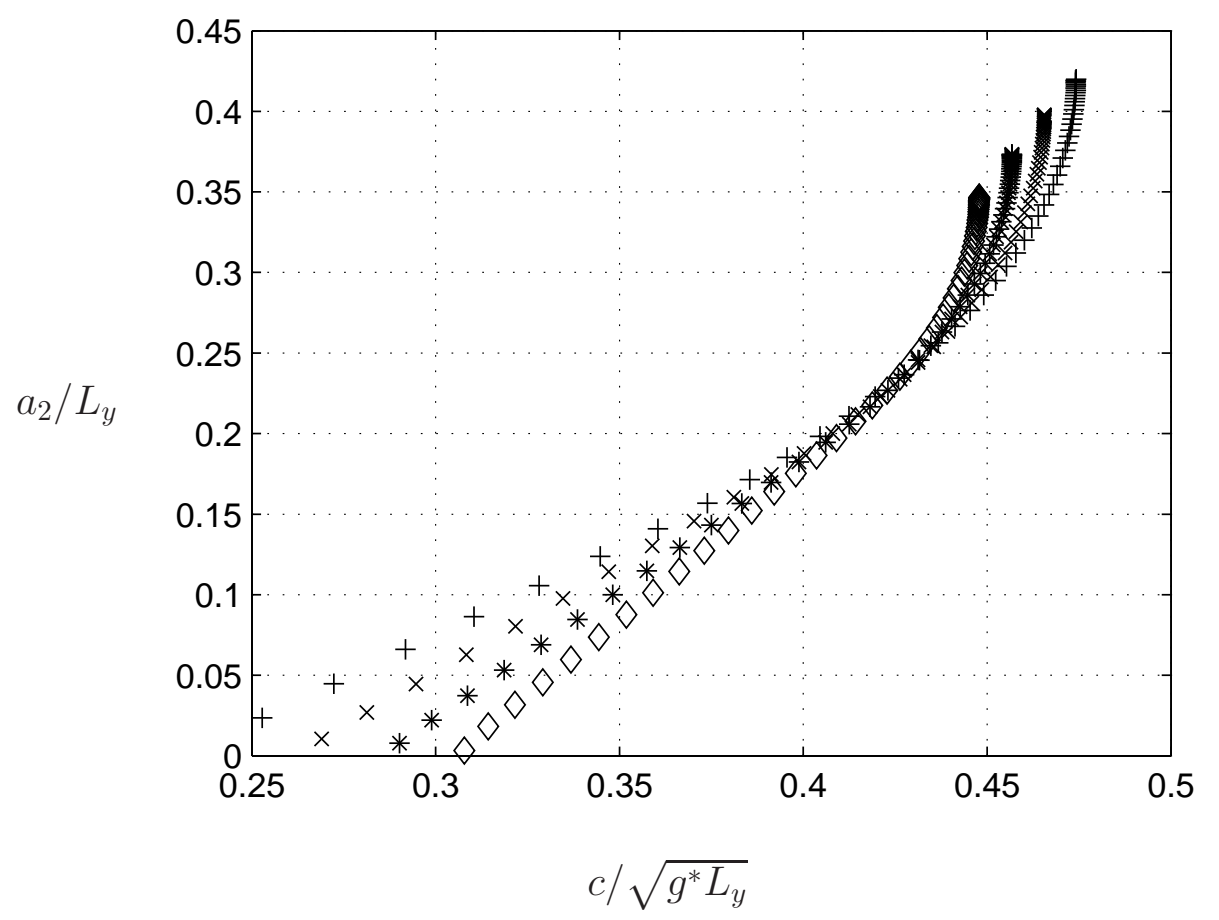

Figure 2: The wave amplitude $a_{2} / L_{y}$ versus the wave speed $c / \sqrt{g^{*} L_{y}}$ for $N_{1}=1$ and $h_{3} /\left(h_{1}+h_{2}\right)=2.33(\diamond), 3(*), 4(\times), 5.66(+)$.

in the top layer $N_{1}$ is equal to the Brunt-Väisälä frequency in the middle layer $N_{2}=1$. The specific case of $N_{1}=1=N_{2}$ is chosen so that comparison with Helfrich \& White ${ }^{23}$ can be made. If $N_{1}=1=N_{2}$ then equation (7) implies that

$$
\Delta \rho=-\frac{\rho_{0} \Delta y}{g}
$$

where $\Delta \rho$ is the change in density over the top two layers and $\Delta y=h_{1}+h_{2}$. In this case the reduced gravity $g^{*}=g \Delta \rho / \rho_{0}=\Delta y$ and wave speed can be non-dimensionalised by $\sqrt{g^{*} L_{y}}$ in keeping with Helfrich \& White. ${ }^{23}$ As expected, figure 2 shows that the wave amplitude increases with increasing wave speed and moreover comparison with figure 8 of Helfrich \& White $^{23}$ shows that the conjugate limit is reached at high amplitudes.

In figure 3, (a) the core height, (b) the half core length, (c) the difference in the rootmean-square of the buoyancy field in the positive and negative halves of the computational 

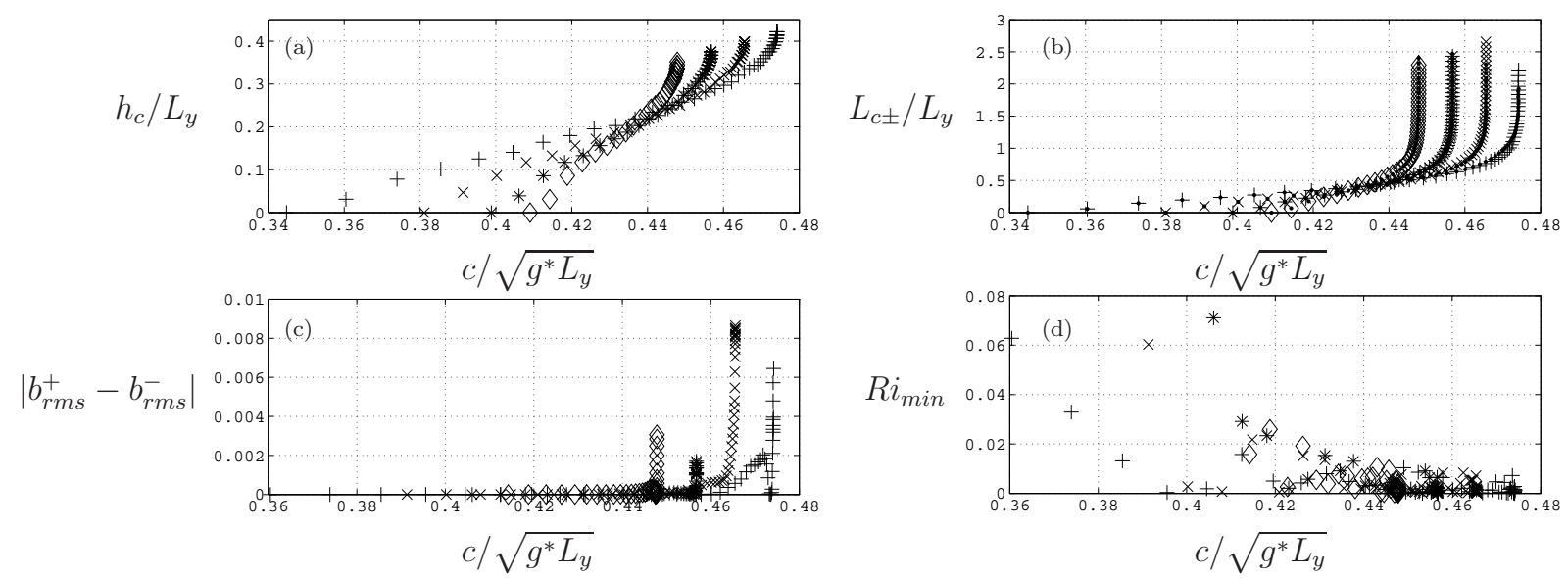

Figure 3: (a) Core height $h_{c} / L_{y}$, (b) half core length $L_{c \pm} / L_{y}$, (c) difference in the root mean square of the buoyancy field in the positive and negative halves of the computational domain $\left|b_{r m s}^{+}-b_{r m s}^{-}\right|$and (d) minimum Richardson number in the computational domain $R i_{m i n}$ versus the wave speed $c / \sqrt{g^{*} L_{y}}$ for the same data set as in figure 2 .

domain and $(\mathrm{d})$ the minimum Richardson number $\left(R i_{\text {min }}=\min \left(b_{y} / \zeta^{2}\right)\right)$ in the computational domain versus wave speed are given respectively, for the same data set as in figure 2 once a core is present $(u>c)$. In figure 3 (b) two lengths are displayed, namely $L_{c \pm}$, where a plus or minus sign indicate the length in the positive and negative halves of the domain respectively (see figure 1). Markers indicate $L_{c+}$ and dots indicate $L_{c-}$. Note that little or no difference can be seen by eye between $L_{c+}$ and $L_{c-}$ until the conjugate limit is approached. At this stage, most noticeably for $h_{3} /\left(h_{1}+h_{2}\right)=4(\times)$ and $5.66(+)$, dots do not directly lie over marker centers. Similarly, figure 3 (c) shows that the difference in the root-mean-square of the buoyancy field in the positive and negative halves of the computational domain is negligible until the wave speed $c / \sqrt{g^{*} L_{y}}$ is at a value corresponding to the large amplitude states that approach the conjugate limit, c.f. figure 2. It is interesting to note that asymmetry was seen in the full computational data set investigated as soon as a core was present. However, the extent of the asymmetry was negligible (of the order of a numerical grid size) until the conjugate limit was approached at which point the asymmetry became more pronounced.

Figures 2, 3 (a) and 3 (b) can be compared with figure 8 of Helfrich \& White. ${ }^{23}$ Al- 
though an exact quantitative comparison cannot be made (as an exact match between the ambient density stratifications has not been made) excellent qualitative agreement can be seen between the model presented here and the stagnant core model of Helfrich \& White 23 (solid line in their figure 8). Note that the stagnant core model of Helfrich \& White, ${ }^{23}$ which follows Turkington et al., ${ }^{30}$ was unable to reach the conjugate state limit. The reason for the failure of the numerical code was unclear but thought to be due to the emergence of low Richardson numbers. ${ }^{23}$ Figure 3 (d) (and later on figure 14) indicate that the model presented here is not limited by low Richardson number. Helfrich \& White $^{23}$ do not note any asymmetry in their steady states. However, the asymmetry seen in the model presented here is negligible until conjugate states are approached so the fact that Helfrich \& White ${ }^{23}$ do not note any asymmetry is not surprising.

For completeness, figure 4 is included to show how (a) the wave amplitude, (b) the core height and (c) the core half length vary with wave speed when the layer thicknesses in the background stratification are held constant and the Brunt-Väisälä frequency in the top layer $N_{1}$ is varied. In these cases the wave speed is non-dimensionalised by the linear long wave speed $c_{0}$, as non-dimensionalisation by $\sqrt{g^{*} L_{y}}$ is not possible since $\Delta \rho$ is not constant over the top two layers. For reference, the data set marked by a cross $(\times)$ in figure 4 is for the same undisturbed background stratification as the data set marked by a cross $(\times)$ in figures 2 and 3. Figure 4 shows that for a given wave speed, as the strength of the Brunt-Väisälä frequency in the top layer $\left(N_{1}\right)$ increases the wave amplitude and core dimensions decrease.

\section{B. Unsteady behavior}

In this section, the evolution of a steady state ISW taken from the data set marked by a $\operatorname{cross}(\times)$ in Sec. IV A is presented. Recall that in this case the undisturbed background stratification is such that $N_{1}=1=N_{2}, N_{3}=0$, and $h_{3} /\left(h_{1}+h_{2}\right)=4$. In all figures the wave propagates from left to right and successive plots are at times $t=0,25,50,75$ and 100, 

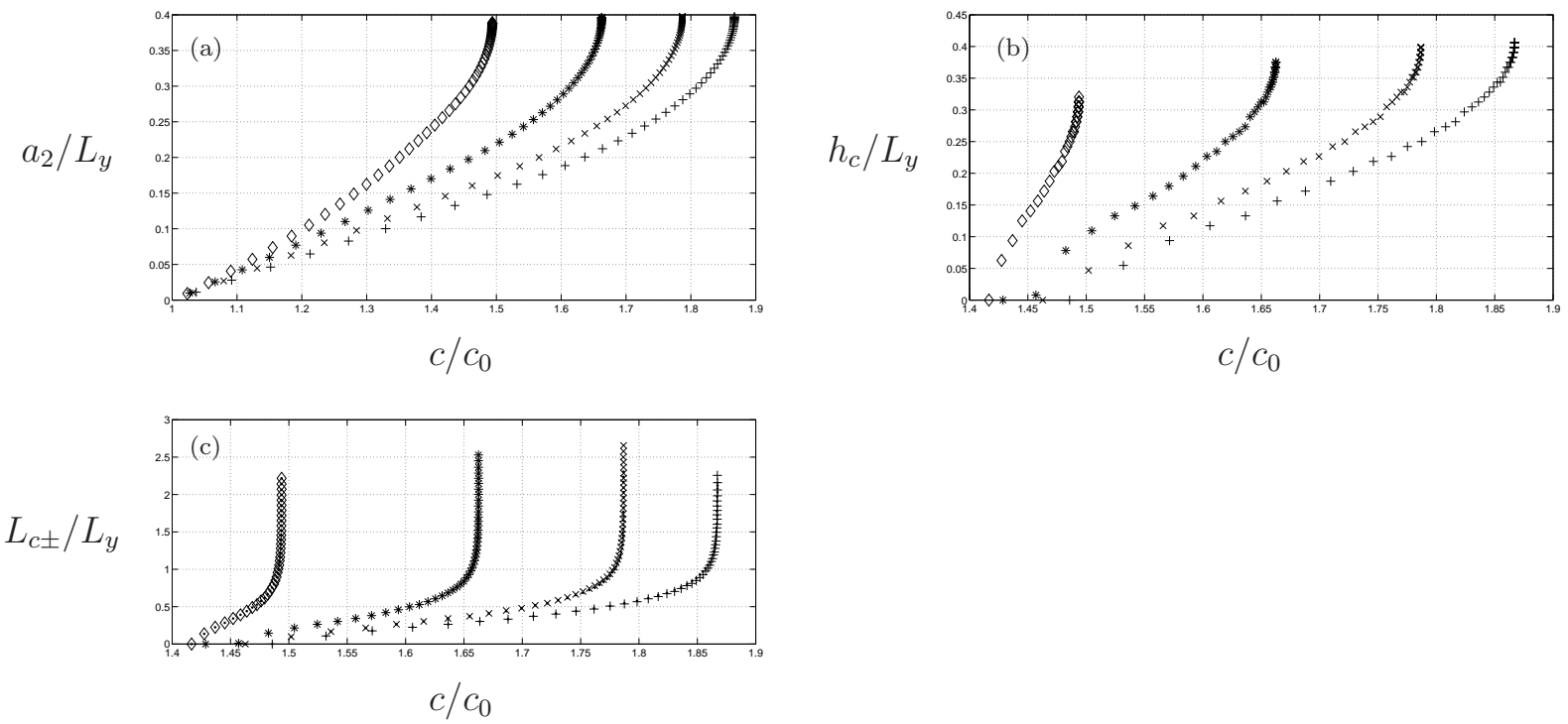

Figure 4: (a) Wave amplitude $a_{2} / L_{y}$, (b) core height $h_{c} / L_{y}$ and (c) core half length $L_{c \pm} / L_{y}$ versus the wave speed $c / c_{0}$ for $h_{2} /\left(h_{1}+h_{2}\right)=0.25, h_{3} /\left(h_{1}+h_{2}\right)=4$ and $N_{1}=0.5(\diamond), 0.75$ $(*), 1(\times), 1.25(+)$.

unless stated otherwise, with time increasing downwards. In figures 5 and 6 , the evolution of the buoyancy field $b$ and the vorticity field $\zeta$ for a wave of amplitude $a_{2} / L_{y}=0.175$ are given respectively. This is the smallest amplitude at which a core forms for the given stratification. The buoyancy field appears to be stable throughout the simulation while the vorticity field exhibits a very small disturbance close to the rear stagnation point at the top of the domain (see panels at $t=50,75$ and 100). To aid visualization a zoomed image of the vorticity field in the core region is presented in figure 7 . Note that the core region (where the vorticity is set to zero via equation (12)) can clearly be seen in the first panel $(t=0)$ of all vorticity plots. The corresponding buoyancy plots are given in the same aspect ratio so that direct comparison can be made if need be.

Figures 8 and 9 show the evolution of the buoyancy field $b$ and the vorticity field $\zeta$, respectively, for a wave at a larger amplitude of $a_{2} / L_{y}=0.319$. Note that the colour scale for vorticity in figure 9 is different from that in figures 6 and 7 . The buoyancy field appears to be stable throughout the simulation while the vorticity field exhibits small-scale disturbances 


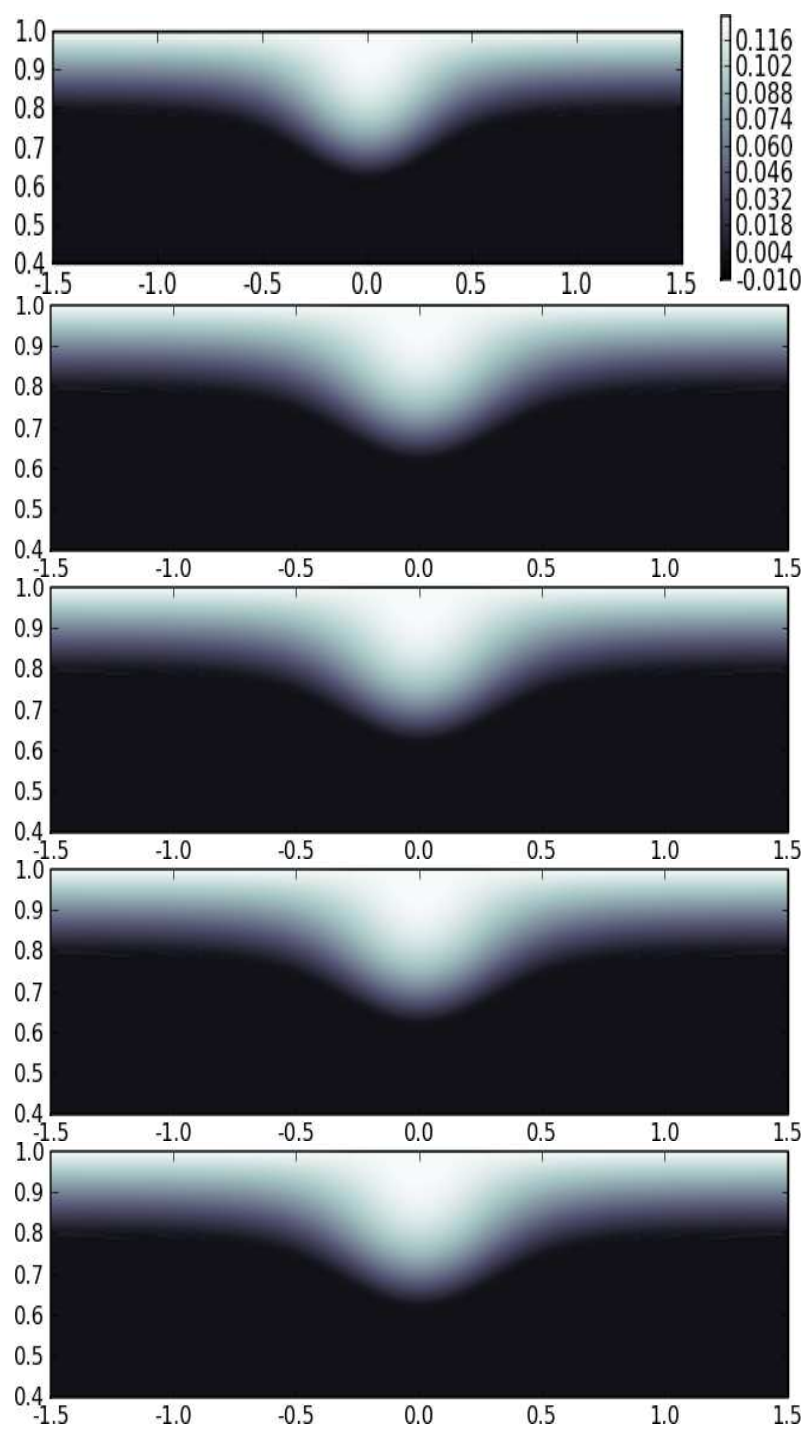

Figure 5: Sequence showing the evolution of the buoyancy field, $b$, for an ISW with, $a_{2} / L_{y}=$ $0.175, N_{1}=1=N_{2}, N_{3}=0$, and $h_{3} /\left(h_{1}+h_{2}\right)=4$. Successive plots are at times $t=$ $0,25,50,75,100$ with time increasing downwards. $\left(x, y / L_{y}\right) \in[-1.5,1.5] \times[0.4,1.0]$. See complementary online movie 1 . 

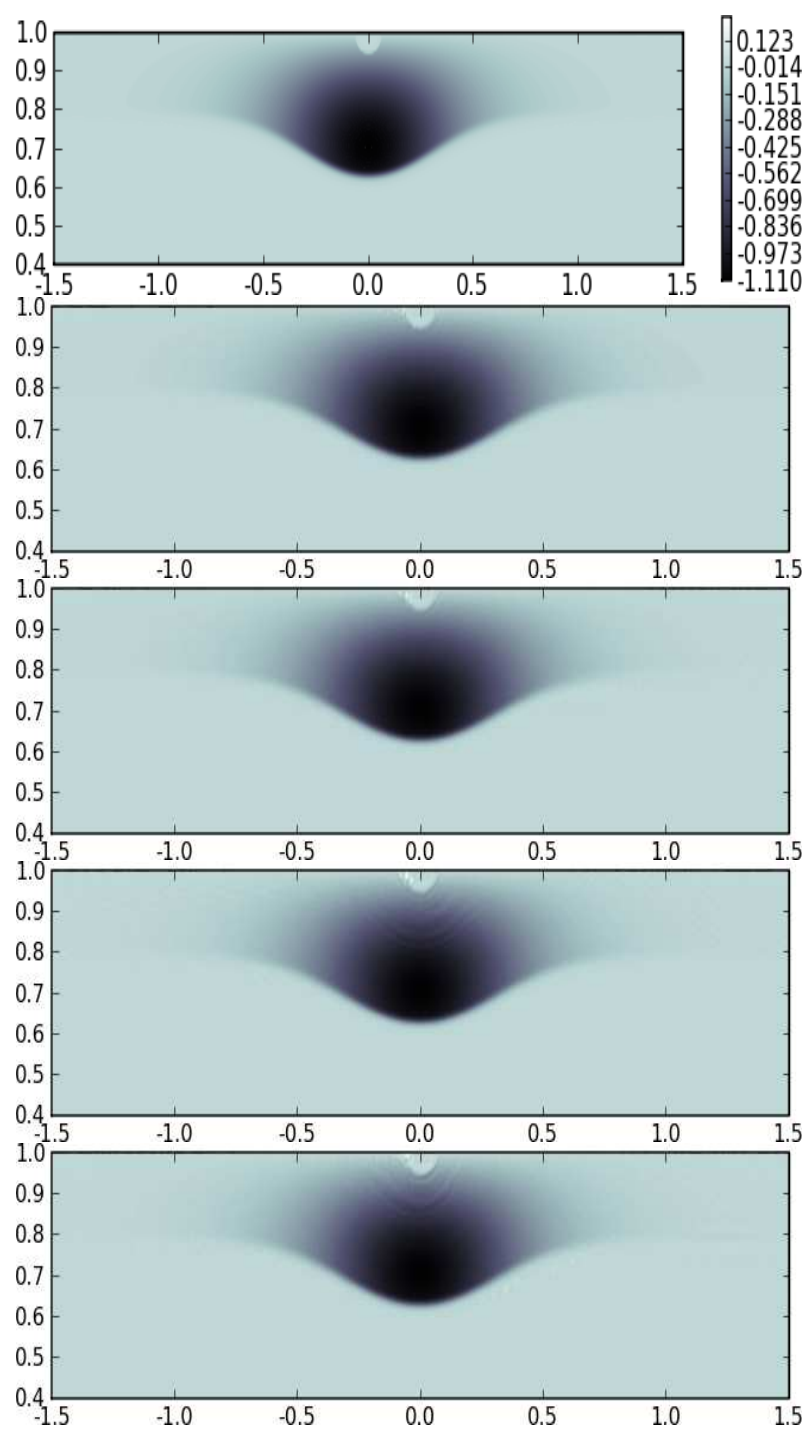

Figure 6: Sequence showing the evolution of the vorticity field, $\zeta$, for the same ISW as in figure 5. See complementary online movie 2. 


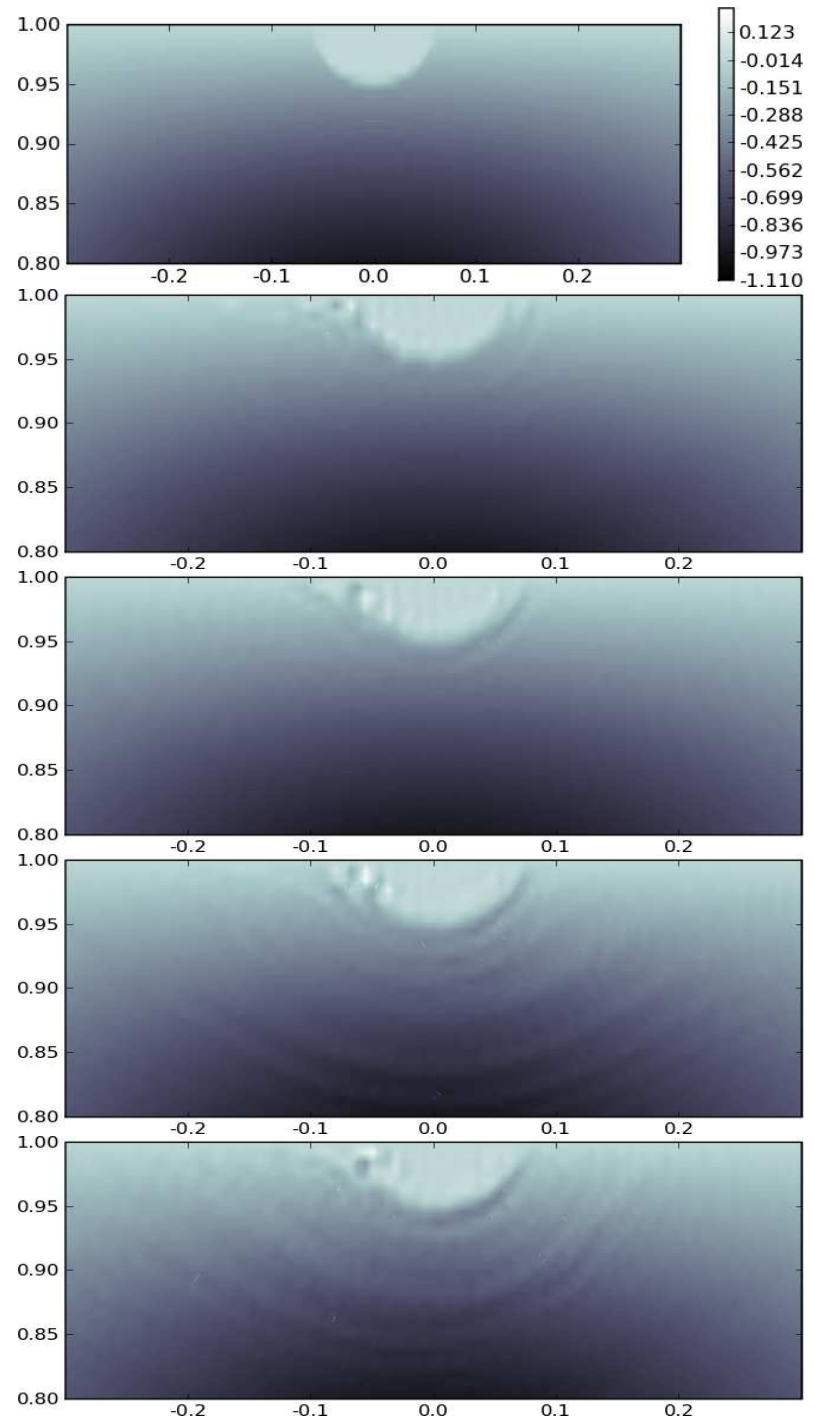

Figure 7: Sequence showing the evolution of the vorticity field, $\zeta$, for the same ISW as in figure 6 over a zoomed in region $\left(x, y / L_{y}\right) \in[-0.3,0.3] \times[0.8,1.0]$. See complementary online movie 3. 


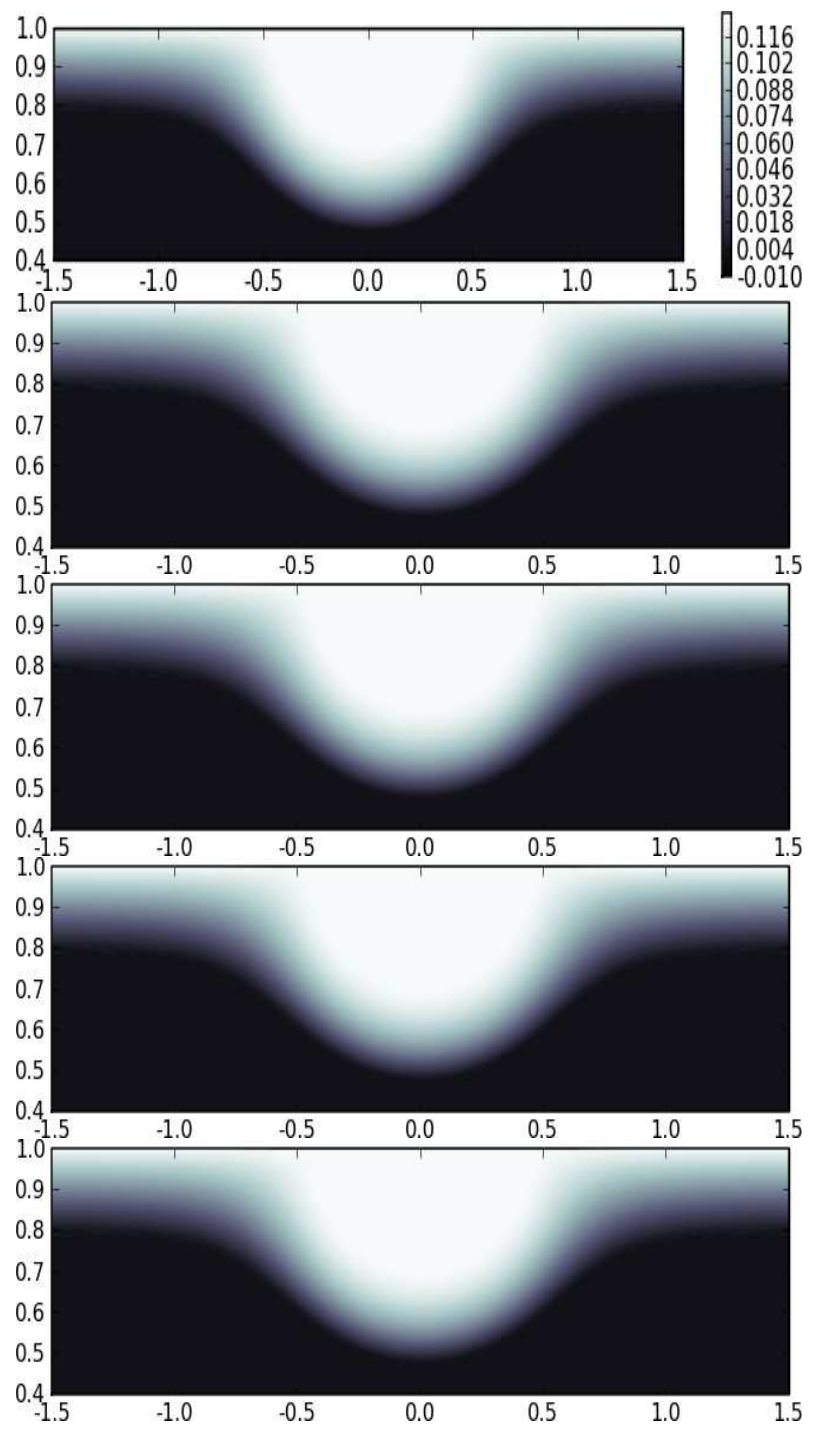

Figure 8: Sequence showing the evolution of the buoyancy field, $b$, for an ISW with, $a_{2} / L_{y}=$ 0.319. All other variables are the same as in figure 5. See complementary online movie 4. 

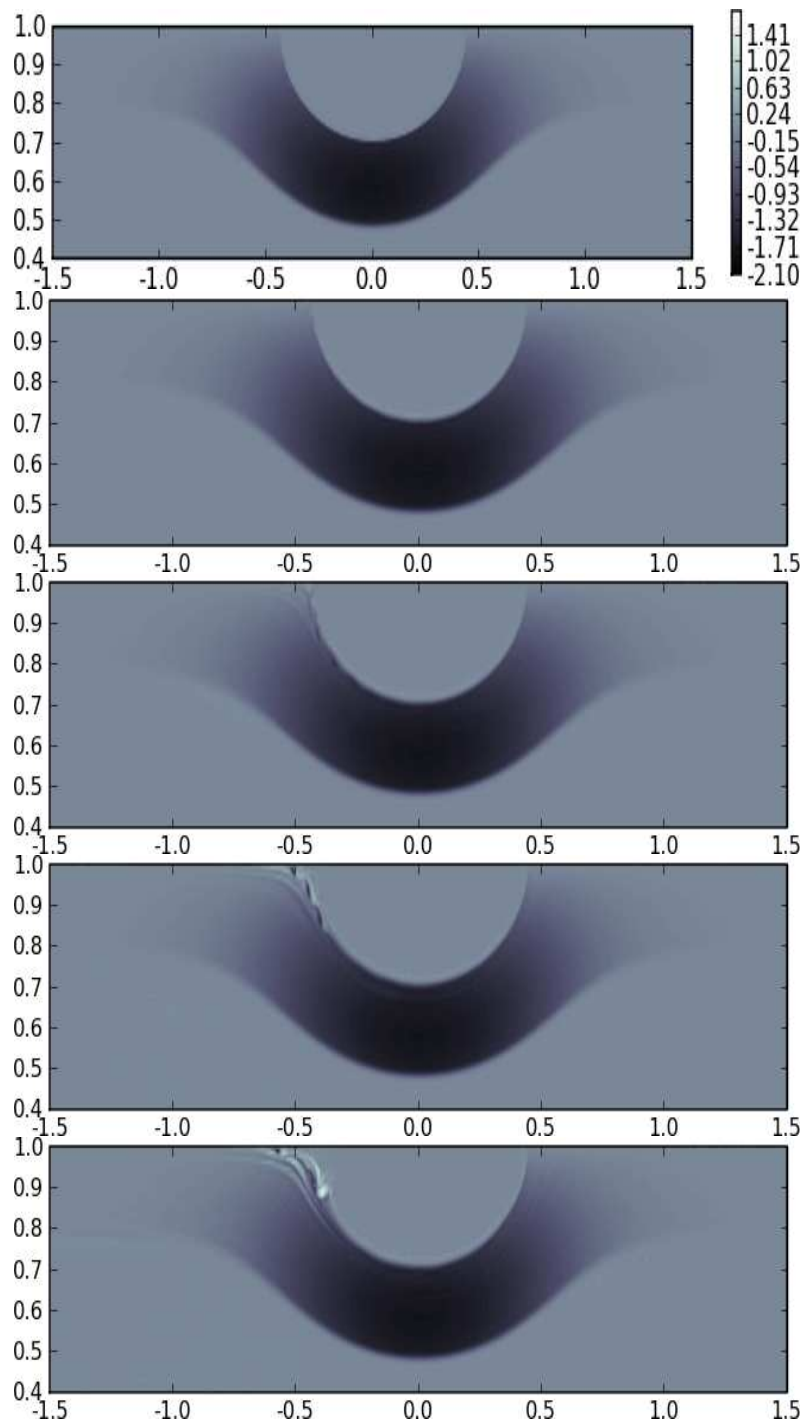

Figure 9: Sequence showing the evolution of the vorticity field, $\zeta$, for the same ISW as in figure 8. See complementary online movie 5. 
on the interface between the core and the pycnocline in the rear of the wave (see panel at $t=50)$. The disturbances appear to take the form of small Kelvin-Helmholtz billows by $t=75$ and the subsequent behavior of these billows at the rear stagnation point can be seen in the panel at $t=100$.

Finally, figures 10 and 11 show the evolution of the buoyancy field $b$ and the vorticity field $\zeta$, respectively, for a wave at a yet larger amplitude of $a_{2} / L_{y}=0.38$. At this amplitude the wave is much broader and flatter than in the smaller amplitude cases (figures 5 to 9 ). This is expected since the conjugate limit is approached at this amplitude (see the data set marked by a cross $(\times)$ in figure 2 at $\left.a_{2} / L_{y}=0.38\right)$. In this case very small disturbances in the buoyancy field occur but are difficult to see over the range of buoyancy values displayed in figure 10. Figure 12 displays $b \in[0.9,0.13]$ and disturbance along the interface between the core and pycnocline region in the tail of the wave can be seen (see panels at $t=50,75$ and 100 in figure 12). The vorticity field (figure 11) shows coherent Kelvin-Helmholtz billows forming on the interface between the core and the pycnocline just ahead of the wave trough (see panel at $t=50)$. The billows subsequently grow $(t=75)$ and on interaction with the rear stagnation point at the top of the domain start to become more energetic $(t=100)$. Note that at this stage the billows loose coherency. Some are swept downstream of the wave along the top of the domain while others are swept upstream along the core boundary. To aid visualization a zoomed image of the vorticity field, for $t=50,63,75,88$ and 100 is presented in figure 13. Note that the colour scale for vorticity in figures 11 and 13 is different from that in figures 6,7 and 9 .

The evolution of the buoyancy and vorticity fields presented in figures 5 to 13 is typical of what was seen in all cases investigated. As soon as a core is formed instability in the vorticity field, albeit small, is seen close to the rear stagnation point (unstable streamlines at the rear stagnation point were noted in King et al. ${ }^{24}$ as being generic, see their figure 13). For higher amplitude states, instability in the vorticity field takes the form of Kelvin- 


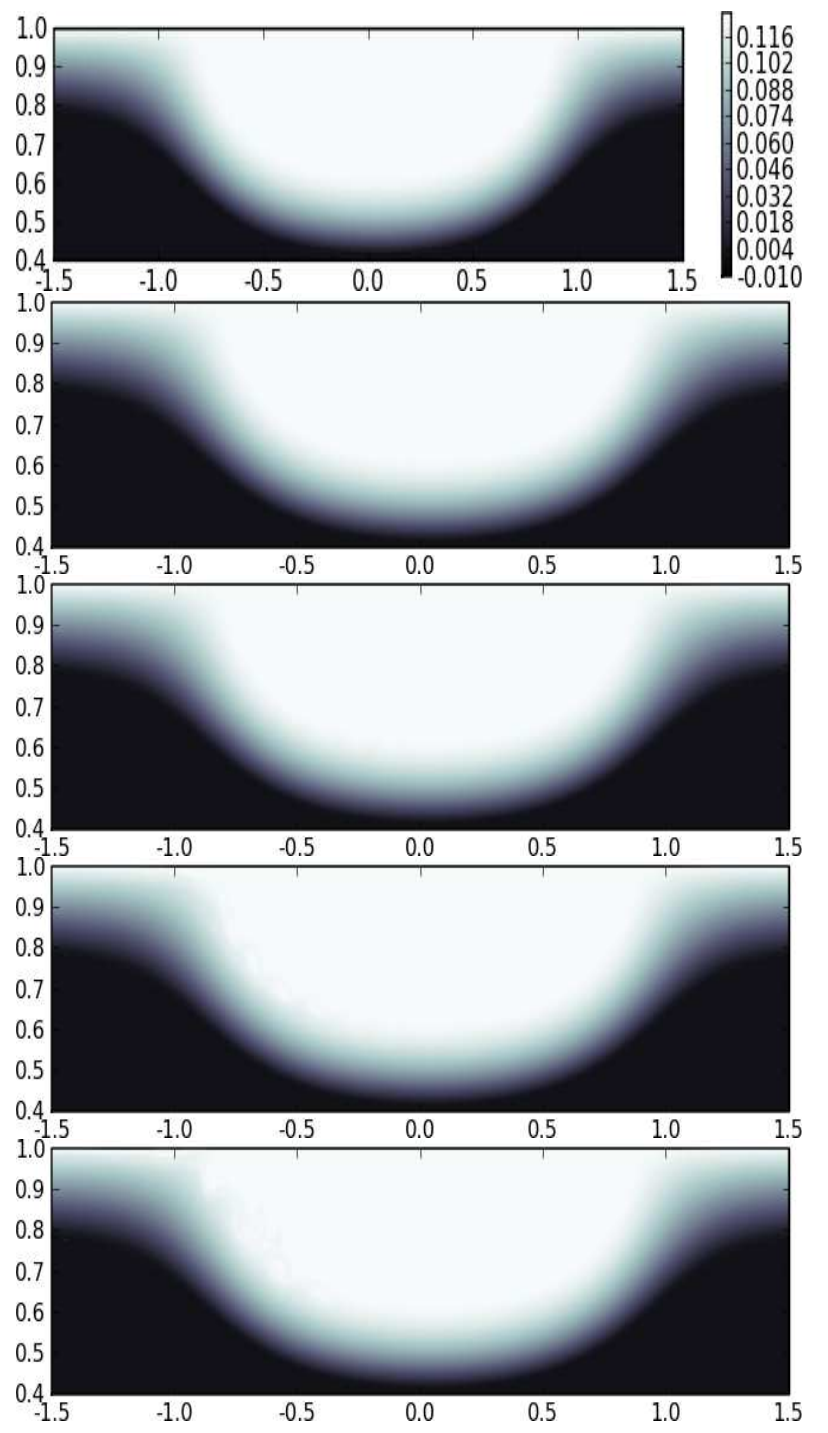

Figure 10: Sequence showing the evolution of the buoyancy field, $b$, for an ISW with, $a_{2} / L_{y}=$ 0.38. All other variables are the same as in figure 5. See complementary online movie 6 . 

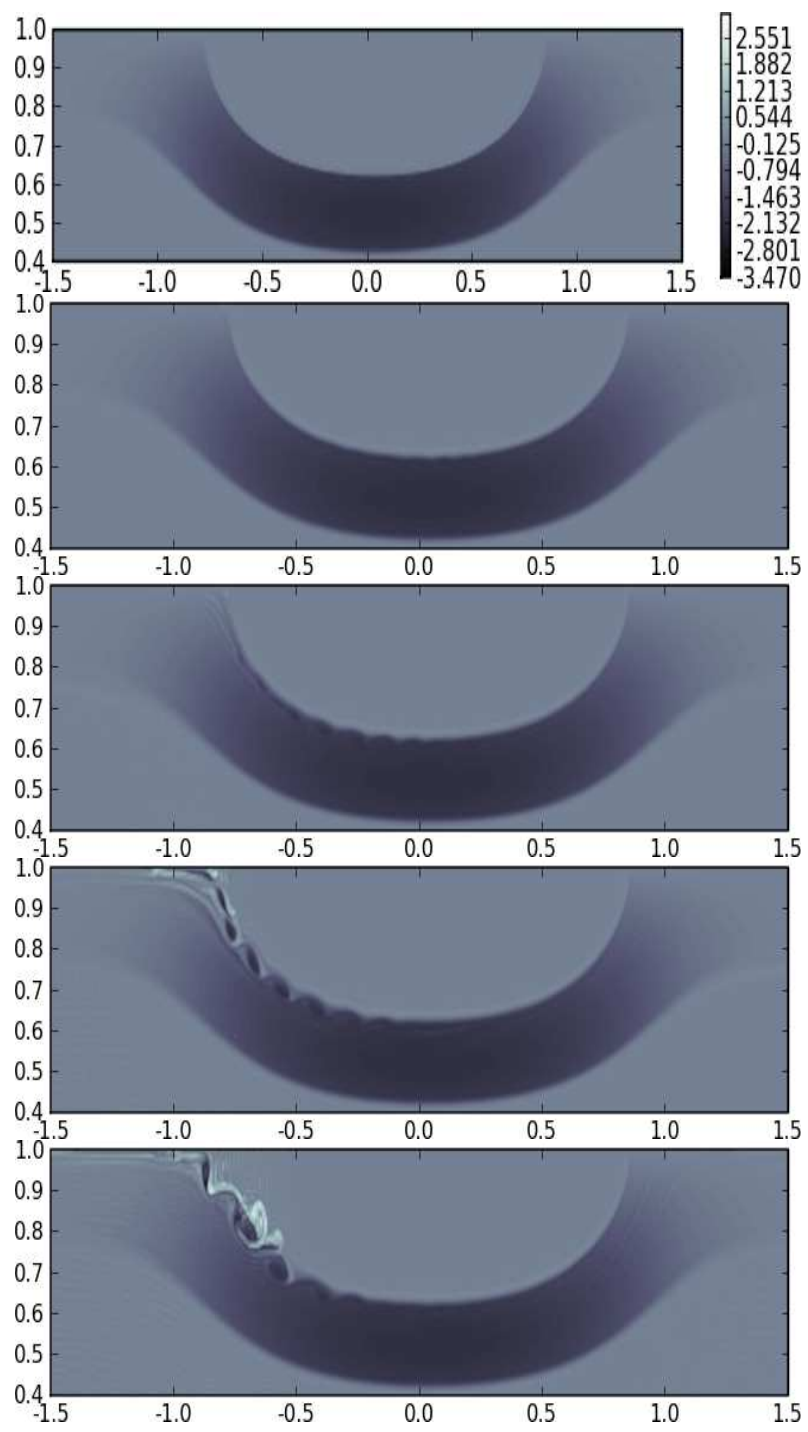

Figure 11: Sequence showing the evolution of the vorticity field, $\zeta$, for the same ISW as in figure 10. See complementary online movie 7. 

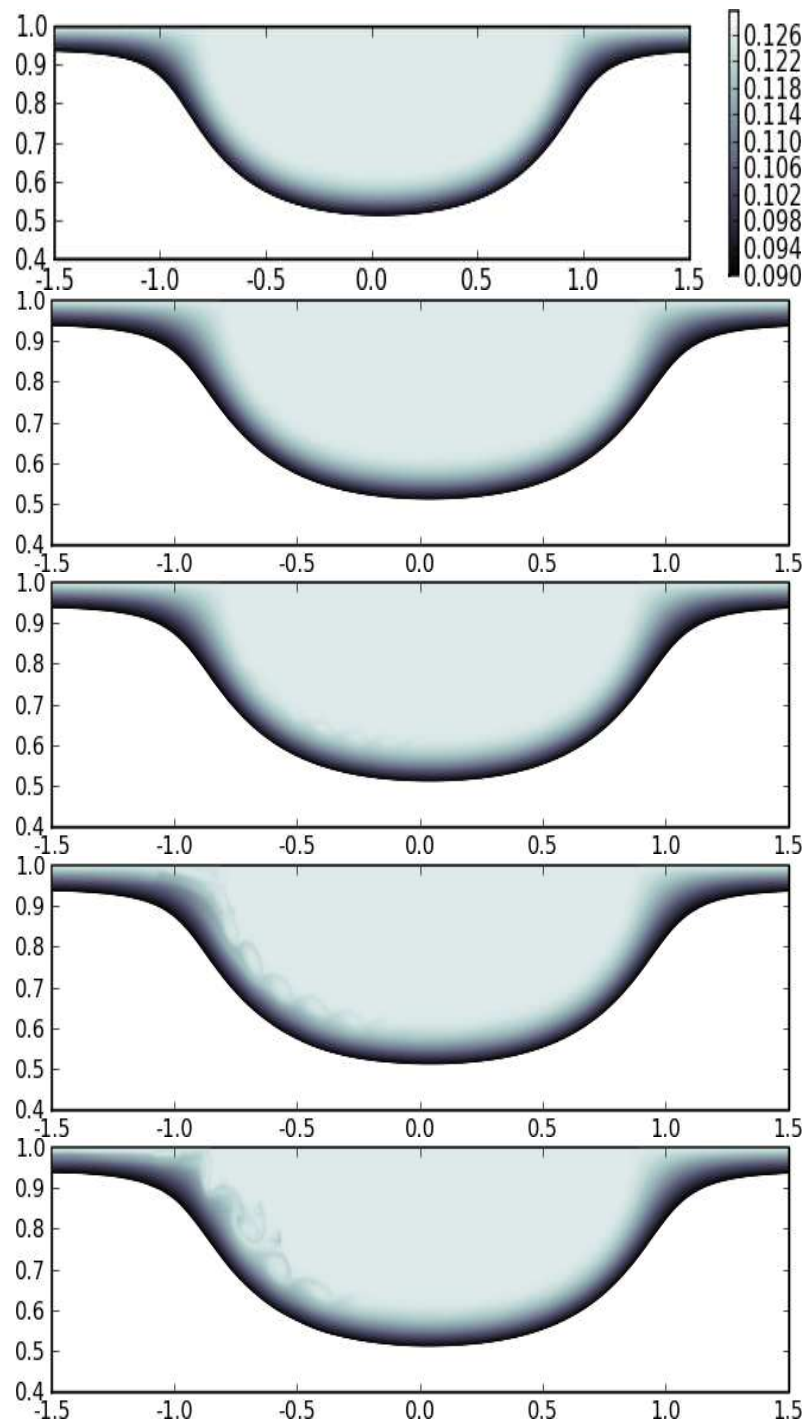

Figure 12: Sequence showing the evolution of the buoyancy field, $b$, for the same ISW as in figure 10 but for a limited range of $b \in[0.09,0.13]$. See complementary online movie 8 . 


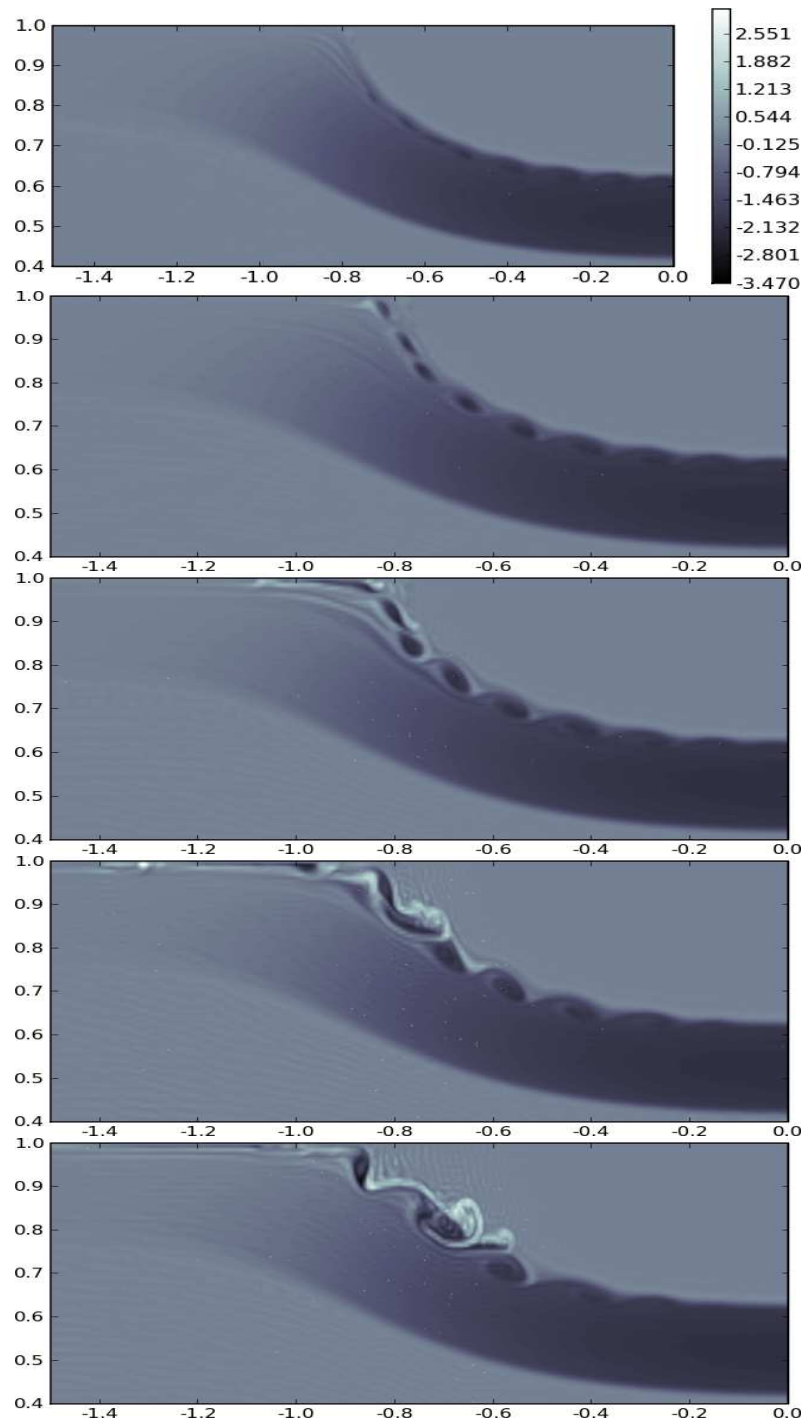

Figure 13: Sequence showing the evolution of the vorticity field, $\zeta$, for the same ISW as in figure 10 over a zoomed in region $\left(x, y / L_{y}\right) \in[-1.5,0.0] \times[0.4,1.0]$. Successive plots are at times $t=50,63,75,88,100$ with time increasing downwards. See complementary online movie 9 . 
Helmholtz billows on the interface between the core and the pycnocline. The billows are coherent and subsequently grow. At the rear stagnation point the billows typically lose coherency and some are swept downstream of the wave while others are carried upstream tracing the boundary between the core and the pycnocline, see figure 13 . If the instability in the vorticity field is energetic enough, disturbances in the buoyancy field can be seen also, see figure 12. This suggests that in such instances, fluid from outside of the core can be entrained into the core region and vice versa. Occurrence of Kelvin-Helmholtz billows is due to the sharp change in the vorticity field at the boundary between the core and the pycnocline, see equation (12) and figure 14 below. At yet higher amplitudes the waves broaden and flatten and a series of Kelvin-Helmholtz billows occur on the interface between the core and the pycnocline akin to Kelvin-Helmholtz billows in parallel shear flow.

In figure 14 contour plots of the Richardson number $\left(R_{i}=b_{y} / \zeta^{2}\right)$ in the initial state wave for the three waves that were allowed to evolve in figures 5 to 13 are given. The area inside the closed solid white line has $R i<0.25$ and the area above the upper solid white line indicates the core region. For visualization purposes values of $R i \geq 1$ are all set to a value equal to 1 . The region in which $R i<0.25$ sits directly below the core boundary and has a length equal to that of the core boundary. This generic picture was seen for all waves investigated in which a core was present and is in agreement with figure 7 of Helfrich \& White $^{23}$ and figures 23 and 24 of Carr et al. ${ }^{14}$ (model (11)).

In Helfrich \& White ${ }^{23}$ it was found that the time-dependent evolution of their stagnant core model, for a wave with $c / \sqrt{g^{*} L_{y}}=0.4$ and $a_{2} / L_{y} \approx 0.2$ (see their figures 8(a), 9 and 10) produced a wave that exhibited instability in the core region. The instability originated along the core boundary and took the form of Kelvin-Helmholtz billows. However, the instability was weak and with time the flow restabilized and the resulting wave was only slightly different from the initial wave. Instability was seen in both the density and the velocity fields. In the model presented here, little or no disturbance was seen in the buoyancy field for waves 


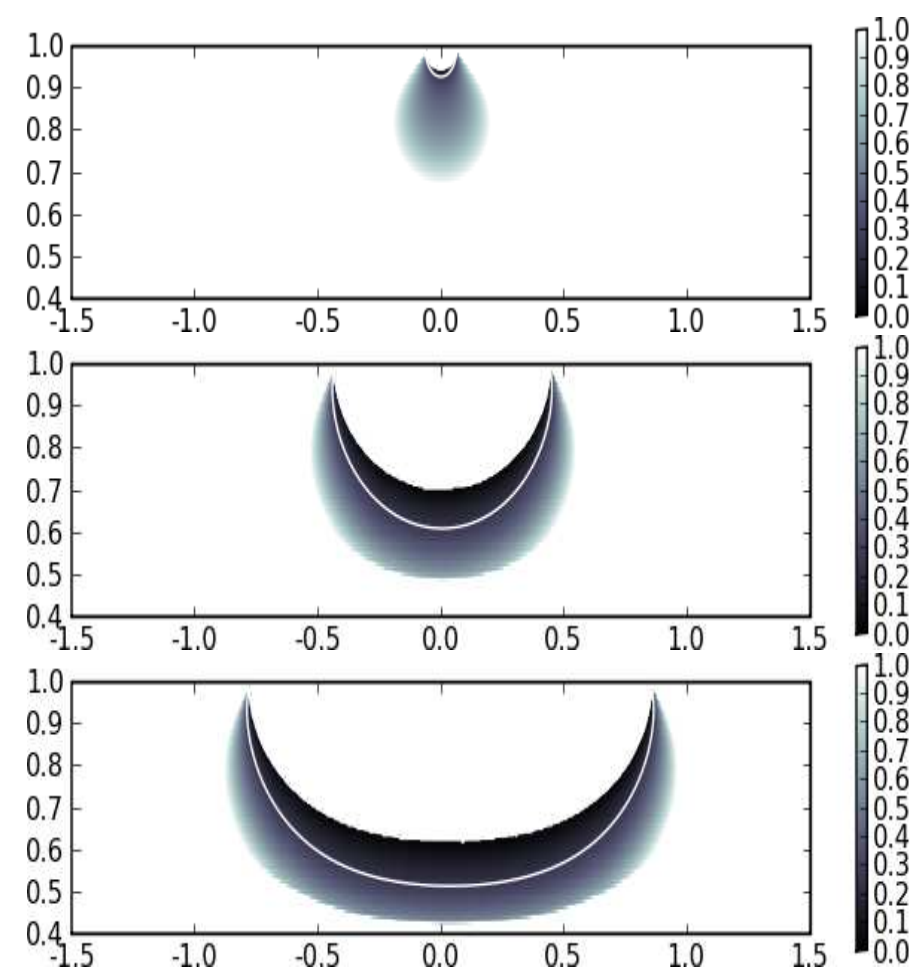

Figure 14: Contour plots of the Richardson number from a steady state ISW which has an undisturbed background stratification identical to that used in figures 5 to 13 . Successive plots are at wave amplitudes of $a_{2} / L_{y}=0.175, a_{2} / L_{y}=0.319$ and $a_{2} / L_{y}=0.38$ (corresponding to figures 5,8 and 10 respectively). The area inside the closed solid white line has $R i<0.25 .\left(x, y / L_{y}\right) \in[-1.5,1.5] \times[0.4,1.0]$. 
at small $\left(a_{2} / L_{y}=0.175\right)$ or moderate $\left(a_{2} / L_{y}=0.319\right)$ amplitudes, see figures 5 and 8 respectively. In particular, note that despite being at a higher amplitude than the wave reported in Helfrich \& White, ${ }^{23}$ figure 8 appears to be much more stable than the evolution of the density field presented in figure 9 of Helfrich \& White. ${ }^{23}$ Evolution of the vorticity field (figure 9) shows good qualitative agreement with Helfrich \& White ${ }^{23}$ but in the model presented here it was found that, in general, once instability was initiated in the wave, the wave did not restabilize. These differences may be due to the way in which diffusion is handled in the two different numerical schemes. Helfrich \& White ${ }^{23}$ employ a finitevolume, second-order projection method which is expected to be more diffusive than the CLAM algorithm employed here (see Dritschel \& Fontane ${ }^{32}$ as well as Fontane \& Dritschel ${ }^{34}$ for detailed comparisons which, in particular address issues of dissipation). In figure 17 of Helfrich \& White, ${ }^{23}$ the evolution of the largest stagnant-core solution they could find was presented $\left(a_{2} / L_{y} \approx 0.363\right)$. In this case the wave was again initially unstable to shear instability localized along the core boundary but unlike in the smaller amplitude case the instability did not weaken. In contrast, the instability grew in strength resulting in the wave speed and size diminishing with time. Comparison of their wave with figures 10, 11 and $12\left(a_{2} / L_{y}=0.38\right)$ again shows good qualitative agreement (in the early stages), though the instability seen here is not as energetic as in Helfrich \& White. ${ }^{23}$ Note that in the computations investigated, the wave speed and size did not diminish with time even in the most unstable cases considered. However, in these cases the simulation duration was restricted because a periodic computational domain was used and as soon as any instability reached the downstream edge of the domain the simulation had to be terminated. Note also that an exact match with the initial stratification in Helfrich \& White ${ }^{23}$ was not made and a strict quantitative comparison of the two therefore cannot be made.

In King et al. ${ }^{24}$ it was claimed that the steady state given by equation (12) was stable when allowed to evolve except for some small-scale disturbance which was attributed to 
numerical fringing. In this paper a much wider parameter space has been investigated and in particular much larger amplitudes have been considered. The results imply that the smallscale disturbance seen in King et al. ${ }^{24}$ is in fact due to shear instability across the transition region from the core to the pycnocline rather than numerical fringing. In addition, the results presented here show that the steady state solutions presented in King et al. ${ }^{24}$ when introduced in the fully nonlinear time dependent numerical code result in a weakly unstable or unstable wave. Figures 5 to 9 clearly show that although the evolution of the buoyancy field is stable at small to moderate amplitudes, the evolution of the vorticity field is not. Moreover, at higher amplitudes, figures 10 to 13, show that both the buoyancy and vorticity fields are weakly unstable or unstable.

Soontiens et al. ${ }^{25}$ also noted that in ISWs with trapped cores the density field was typically more stable than the velocity field. They presented a model for trapped core waves in the presence of a background shear flow. When a background shear was present they found that waves with vortex cores could persit for long times in time-dependent simulations and agree well with solutions of steady theory. However, in the absence of background shear they found streamline overturning in the core and the time-dependent simulations yielded unsteady cores which did not match the steady results very well. They found that over very long times, the majority of fluid in the breaking region was flushed out downstream of the wave resulting in a core region that was essentially homogeneous in density but in which the velocity field did not stabilize.

\section{CONCLUSION}

A fully nonlinear time dependent simulation was used to investigate the steady state model presented in King et al. ${ }^{24}$ for ISWs that exhibit trapped cores. To achieve this, a background stratification was chosen such that the Brunt-Väisälä frequency in the top layer was non 
zero. The counterpart case, in which the Brunt-Väisälä frequency in the top layer is zero, is fundamentally different and investigation is found in Carr et al. ${ }^{35}$ The steady state model ${ }^{24}$ assumes that the DJL equation is satisfied both inside and outside of the core region and that the core region is stagnant (zero vorticity) in a frame of reference moving with the wave and of a homogeneous density which matches the density in the ambient fluid at the core boundary. The time dependent simulations showed that the model produced waves with trapped cores that exhibit Kelvin-Helmholtz billows on the interface between the core and the pycnocline. If the instability was energetic enough then disturbance in the buoyancy field could be seen as well as in the vorticity field. In general the buoyancy field was much more stable than the vorticity field and the occurrence of the Kelvin-Helmholtz billows was attributed to the sharp change in the vorticity field at the boundary between the core and the pycnocline. The qualitative behavior of the unsteady ISWs was very like that in the stagnant core model presented by Helfrich \& White. ${ }^{23}$

In Helfrich \& White ${ }^{23}$ a vorticity-streamfunction relation was assumed to hold in the core region while elsewhere the DJL equation was employed. A pressure matching condition was imposed along the core boundary to match the two different flow regimes. The results presented here show that the DJL model of King et al., ${ }^{24}$ while having no physically justification in the core region, essentially produces waves with a similar time dependent behavior to those generated by the pressure matching model of Helfrich \& White. ${ }^{23}$ The model of Helfrich \& White ${ }^{23}$ appears to be limited by small Richardson numbers whereas the model of King et al. ${ }^{24}$ does not. Hence, it can be argued that the model of King et al. ${ }^{24}$ is a sensible alternative to Helfrich \& White ${ }^{23}$ at small Richardson numbers.

In the higher amplitude cases investigated, if the instability in the vorticity field was energetic enough, disturbances in the buoyancy field were also seen (see figures 10, 11 and 12). This suggests that in such instances, exchange of fluid can occur across the core boundary. A similar observation was made in the laboratory by Carr et al. ${ }^{14}$ Therein, ISWs with 
unstable trapped cores were generated using a lock release method. The water column in the main section of the tank was seeded with tracer particles for flow visualization while that behind the lock was left unseeded. The unstable core (in the subsequently generated wave) contained tracer particles. This suggests that ambient fluid must have been entrained into the core region during wave propagation. It is unclear whether entrainment is due to the wave generation mechanism, if it is a characteristic of the wave propagation itself or if it is a combination of the two. Helfrich \& White ${ }^{23}$ modelled the lock release system of Carr et al. ${ }^{14}$ and found that the ensuing instability was significantly different to their stagnant core model. Hence a comparison of laboratory observations with the results presented here should be made with caution. However, entrainment of ambient fluid into the core appears to take place in (i) the model presented here, (ii) the model of Helfrich \& White $^{23}$ and (iii) the laboratory observations of Carr et al. ${ }^{14}$ Exchange of fluid between the core and ambient surroundings has significant implications in the field for mass and momentum transfer. Hence, a further laboratory study has begun in which detailed density measurements are taken in the core region in the hope that they may improve our physical understanding of the processes involved.

As far as the authors are aware there are no field observations of ISWs exhibiting instability exactly like that presented here. This is not surprising, as recent numerical ${ }^{31,35}$ and laboratory ${ }^{14}$ studies have shown that the onset and type of instability in an ISW is sensitive to the ambient stratification. The background stratification investigated here is idealized and does not incorporate any background flow which is inevitable in field observations. Moreover, a lack of resolution in presently available field data ${ }^{8-12}$ makes it difficult to determine the exact nature of instability in ISWs with trapped cores. There is a pressing need for more detailed field studies to resolve some of the issues discussed above. 


\section{ACKNOWLEDGMENTS}

The authors are grateful for support from the UK Engineering and Physical Sciences Research Council under its Mathematical Sciences Programme (grant number EP/F030622/1). The authors would like to thank Marek Stastna whose constructive criticisms have led to improvements in the paper. The authors would also like to thank Karl Helfrich for fruitful discussion of the work.

\section{References}

[1] J. R. Apel, L. A. Ostrovsky, Y. A. Stepanyants and J. F. Lynch, "Internal solitons in the ocean," Techinical Report, Woods Hole Oceanog. Inst. (2006).

[2] J. Grue, "Waves in geophysical fluids-tsunamis, rogue waves, internal waves and internal tides", Springer, First Edition, pages 205-270, (2006).

[3] K. R. Helfrich and W. K. Melville, "Long nonlinear internal waves," Annu. Rev. Fluid. Mech. 38, 395 (2006).

[4] J. R. Apel, L. A. Ostrovsky, Y. A. Stepanyants and J. F. Lynch, "Internal solitons in the ocean and their effect on underwater sound," J. Acoust. Soc. Am. 121, 695 (2007)

[5] T. P. Stanton and L. A. Ostrovsky, "Observations of highly nonlinear internal solitons over the continental shelf," Geophys. Res. Lett. 25(14), 2695 (1998).

[6] M. H. Orr and P. C. Mignerey, "Nonlinear internal waves in the South China Sea: Observation of the conversion of a depression internal waves to elevation internal waves," J. Geophys. Res. 108, 3064 (2003).

[7] T. F. Duda, J. F. Lynch, J. D. Irish, R. C. Beardsley, S. R. Ramp, C.-S. Chiu, T. Y. Tang and Y. J. Yang, "Internal tide and nonlinear internal wave behavior at the 
continental slope in the northern South China Sea", IEEE J. Oceanic Eng. 29, 1105 (2004).

[8] J. M. Klymak and J. N. Moum, "Internal solitary waves of elevation advancing on a shoaling shelf," Geophys. Res. Lett. 30, 2045 (2003).

[9] A. Scotti and J. Pineda, "Observation of very large and steep internal waves of elevation near the Massachusetts coast," Geophys. Res. Lett. 31, L22307 (2004).

[10] R. H. Clarke, R. K. Smith and D. G. Reid, "The Morning Glory of the Gulf of Carpentaria: An atmospheric undular bore," Mon. Weather Rev. 109, 1726, (1981).

[11] R. J. Doviak and D. R. Christie, "Thunderstorm-generated solitary waves: A wind shear hazard," J. Aircr. 26, 423, (1989).

[12] T. K. Cheung and C. G. Little, "Meterological tower, microbarograph array, and sodar observations of solitary-like waves in the nocturnal boundary layer," J. Atmos. Sci. 47, 2516, (1990).

[13] J. Grue, A. Jensen, P. -O. Rusås, P. -O. and J. K. Sveen, "Breaking and broadening of internal solitary waves," J. Fluid Mech. 413, 181 (2000).

[14] M. Carr, D. Fructus, J. Grue, A. Jensen and P. A. Davies, "Convectively induced shear instability in large amplitude internal solitary waves," Phys. Fluids. 20, 12660 (2008).

[15] R. E. Davis and A. Acrivos, "Solitary internal waves in deep water," J. Fluid Mech. 29(3), 593 (1967).

[16] O. G. Derzho and R. Grimshaw, "Solitary waves with a vortex core in a shallow layer of stratified fluid," Phys. Fluids. 9, 3378 (1997). 
[17] D. J. Brown and D. R. Christie, "Fully nonlinear solitary waves in continuously stratified incompressible Boussinesq fluids," Phys. Fluids. 10(10), 2569 (1998).

[18] A. Aigner, D. Broutman and R. Grimshaw, "Numerical simulations of internal solitary waves with vortex cores," Fluid Dyn. Res. 25, 315 (1999).

[19] M. Stastna and K. G. Lamb, "Internal solitary-like waves with recirculating cores," Proc. 5th ISSF, ed. G. Lawerence, N. Yonemitsu, R. Pieters. 821 (2000).

[20] K. G. Lamb, "A numerical investigation of solitary internal waves with trapped cores formed via shoaling," J. Fluid Mech. 451, 109 (2002).

[21] K. G. Lamb and K. P. Wilkie, "Conjugate flows for waves with trapped cores," Phys. Fluids. 16(12), 4685 (2004).

[22] D. Fructus and J. Grue, "Fully nonlinear solitary waves in a layered stratified fluid," J. Fluid Mech. 505, 323 (2004).

[23] K. R. Helfrich and B. L. White, "A model for large-amplitude internal solitary waves with trapped cores," Nonlin. Processes Geophys. 17, 303 (2010).

[24] S. E. King, M. Carr and D. G. Dritschel, "The steady state form of large amplitude internal solitary waves," J. Fluid Mech. 666, 477 (2010).

[25] N. Soontiens, C. Subich and M. Stastna, "Numerical simulation of supercritical trapped internal waves over topography," Phys. Fluids. 22, 116605 (2011).

[26] L. Dubreil-Jacotin, "Sur la determination rigoureuse des ondes permantentes periodiques d'amplitude finite," J. Math. Pure Appl. 13, 217 (1934).

[27] R. R. Long, "Some aspects of the flow of stratified fluids, I.A theoretical investigation," Tellus, 5, 42, (1953). 
[28] J. Pineda, "Circulation and larval distribution in internal tidal bore warm fronts, " Limnol. Oceanogr. 44, 1400, (1999).

[29] K. R. Helfrich and J. Pineda, "Accumulation of particles in propagating fronts," Limnol. Oceanogr. 48, 1509, (2003).

[30] B. Turkington, A. Eydeland and S. Wang, " A computational method for solitary internal waves in a continuously stratified fluid," Stud. Appl. Math. 85, 93 (1991).

[31] K. G. Lamb and D. Farmer, "Instabilities in an internal solitary-like wave on the Oregeon shelf," J. Phys. Oceanogr. 41, 67 (2011).

[32] D. G. Dritschel and J. Fontane, "The combined lagrangian advection method," J. Comput. Phys. 229, 5408 (2010).

[33] D. G. Dritschel and R. K. Scott, "On the simulation of nearly inviscid two-dimensional turbulence," J. Comput. Phys. 228, 2707 (2009).

[34] J. Fontane and D. G. Dritschel, "The HyperCASL algorithm: a new approach to the numerical simulation of geophysical flows," J. Comput. Phys. 228, 6411, (2009).

[35] M. Carr, S. E. King and D. G. Dritschel, "Numerical simulation of shear-induced instabilities in internal solitary waves," J. Fluid Mech. 683, 263 (2011).

[36] C-S. Yih, C-S, "Exact solutions for steady two-dimensional flow of a stratified fluid," J. Fluid Mech. 9, 161 (1960).

[37] D. G. Dritschel and M. H. P. Ambaum, "A contour-advective semi-Lagrangian numerical algorithm for simulating fine-scale conservative dynamical fields," Quart. J. Roy. Meteorol. Soc. 123, 1097 (1997). 


\section{List of Figures}

Figure 1: A schematic diagram of the computational domain (not to scale).

Figure 2: The wave amplitude $a_{2} / L_{y}$ versus the wave speed $c / \sqrt{g^{*} L_{y}}$ for $N_{1}=1$ and $h_{3} /\left(h_{1}+h_{2}\right)=2.33(\diamond), 3(*), 4(\times), 5.66(+)$.

Figure 3: (a) Core height $h_{c} / L_{y}$, (b) half core length $L_{c \pm} / L_{y}$, (c) difference in the root mean square of the buoyancy field in the positive and negative halves of the computational domain $\left|b_{r m s}^{+}-b_{r m s}^{-}\right|$and (d) minimum Richardson number in the computational domain $R i_{\text {min }}$ versus the wave speed $c / \sqrt{g^{*} L_{y}}$ for the same data set as in figure 2 .

Figure 4:(a) Wave amplitude $a_{2} / L_{y}$, (b) core height $h_{c} / L_{y}$ and (c) core half length $L_{c \pm} / L_{y}$ versus the wave speed $c / c_{0}$ for $h_{2} /\left(h_{1}+h_{2}\right)=0.25, h_{3} /\left(h_{1}+h_{2}\right)=4$ and $N_{1}=0.5(\diamond), 0.75$ $(*), 1(\times), 1.25(+)$.

Figure 5: Sequence showing the evolution of the buoyancy field, $b$, for an ISW with, $a_{2} / L_{y}=0.175, N_{1}=1=N_{2}, N_{3}=0$, and $h_{3} /\left(h_{1}+h_{2}\right)=4$. Successive plots are at times $t=0,25,50,75,100$ with time increasing downwards. $\left(x, y / L_{y}\right) \in[-1.5,1.5] \times[0.4,1.0]$. See complementary online movie 1 .

Figure 6: Sequence showing the evolution of the vorticity field, $\zeta$, for the same ISW as in figure 5. See complementary online movie 2.

Figure 7: Sequence showing the evolution of the vorticity field, $\zeta$, for the same ISW as in figure 6 over a zoomed in region $\left(x, y / L_{y}\right) \in[-0.3,0.3] \times[0.8,1.0]$. See complementary 
online movie 3 .

Figure 8: Sequence showing the evolution of the buoyancy field, $b$, for an ISW with, $a_{2} / L_{y}=0.319$. All other variables are the same as in figure 5. See complementary online movie 4 .

Figure 9: Sequence showing the evolution of the vorticity field, $\zeta$, for the same ISW as in figure 8. See complementary online movie 5 .

Figure 10: Sequence showing the evolution of the buoyancy field, $b$, for an ISW with, $a_{2} / L_{y}=0.38$. All other variables are the same as in figure 5. See complementary online movie 6 .

Figure 11: Sequence showing the evolution of the vorticity field, $\zeta$, for the same ISW as in figure 10. See complementary online movie 7 .

Figure 12: Sequence showing the evolution of the buoyancy field, $b$, for the same ISW as in figure 10 but for a limited range of $b \in[0.09,0.13]$. See complementary online movie 8 .

Figure 13: Sequence showing the evolution of the vorticity field, $\zeta$, for the same ISW as in figure 10 over a zoomed in region $\left(x, y / L_{y}\right) \in[-1.5,0.0] \times[0.4,1.0]$. Successive plots are at times $t=50,63,75,88,100$ with time increasing downwards. See complementary online movie 9.

Figure 14: Contour plots of the Richardson number from a steady state ISW which has an undisturbed background stratification identical to that used in figures 5 to 13 . Successive 
plots are at wave amplitudes of $a_{2} / L_{y}=0.175, a_{2} / L_{y}=0.319$ and $a_{2} / L_{y}=0.38$ (corresponding to figures 5, 8 and 10 respectively). The area inside the closed solid white line has $R i<0.25 .\left(x, y / L_{y}\right) \in[-1.5,1.5] \times[0.4,1.0]$. 